\title{
IS ROTATION A REALLY IMPORTANT PROPERTY FOR THE UNDER- STANDING OF THE EVOLUTION OF MASSIVE STARS?
}

\author{
S.R. Sreenivasan \\ Department of Physics and Astronomy \\ The University of Calgary \\ Calgary, $A B$, T2N 1N4, Canada
}

Massive stars have been observed to possess significant rotational speeds. The frequency distribution shows two peaks one centred around $100 \mathrm{~km} / \mathrm{s}$ and another about $250 \mathrm{~km} / \mathrm{s}$. No star has been observed to rotate at or near the critical speed which balances centrifugal force with gravity. This fact has played a large role in the neglect of rotational effects as being unimportant and dismissed as being very uncertain in the literature. On the other hand, standard models do not explain many of the observed features of stellar evolution in this mass-range. It is also customary to employ crude parameterised forms for significant effects such as those of mass-loss, as well as ad hoc variations in the rates of mass-loss which were originally derived by using statistical fits to observed mass-loss rates over a wide range of masses, effective temperatures and luminosities. Further, unrealistic prescriptions for phenomena that are not properly understood such as convective core overshoot are employed to bring about agreement with observed properties of massive stars that are still not completely secure.

It is shown in this work that even relatively low rotational speeds can cause significant effects to the observable properties and distributions of massive stars. Due to mass and angular momentum loss observed rotational speeds of stars do not indicate either the original speed or the history of the rotational speeds as the stars evolve. One can model self-consistently the rotational effects for stars which have relatively low speeds of the order of 1 to $3 \%$ in the ratio of centrifugal force to gravity and demonstrate that a number of features of massive stars and their evolutionary characteristics can be understood without the use of ad hoc formulations of the effects. In particular, the evolutionary history of the progenitor of SN 1987A, the characteristics of the evolution of the LMC stars as well as the pulsational characteristics of OB stars can be delineated without much stress. When applied to intermediate mass stars rotational effects can again explain many of the observed features that are not explainable on the basis of standard models. Some examples are: Blue Stragglers, Peculiar Red Giants, High Latitude F Supergiants. We conclude that rotational effects are quite important and cannot be neglected.

K. A. van der Hucht and B. Hidayat (eds.),

Wolf-Rayet Stars and Interrelations with Other Massive Stars in Galaxies, 551.

(C) 1991 IAU. Printed in the Netherlands. 